\title{
Rivaroxaban treatment for cancer-associated venous thromboembolism in a patient with heparin-induced thrombocytopenia: a case report
}

\author{
Ke-Jia Le ${ }^{1 \#}$, Yi-Dan Yan ${ }^{1 \#}$, Yang-Xi Liu ${ }^{1}$, Jia-Bo Xu' ${ }^{2}$, Min Cui ${ }^{1}$, Zhi-Chun Gu ${ }^{1}$ \\ ${ }^{1}$ Department of Pharmacy, ${ }^{2}$ Department of Respiration, Renji Hospital, School of Medicine, Shanghai Jiaotong University, Shanghai 200127, China \\ \#These authors contributed equally to this work. \\ Correspondence to: Zhi-Chun Gu, MD. Department of Pharmacy, Renji Hospital, School of Medicine, Shanghai Jiaotong University, Shanghai \\ 200127, China. Email: guzhichun213@163.com.
}

\begin{abstract}
Low molecular weight heparin (LMWH) is the first-line therapy in acute cancer-associated venous thromboembolism (CAT). However, heparin-induced thrombocytopenia (HIT) is a life-threatening adverse drug reaction that occurs in anticoagulation therapy with LMWH. This article reports the case of a 66-year-old Chinese male who received nadroparin 4100IU twice daily for treating CAT. Unfortunately, the epistaxis persisted and the blood count examination revealed serious thrombocytopenia on postoperative day 5. The patient was diagnosed with HIT and thereafter LMWH therapy was replaced with rivaroxaban. During three months follow-up, the patient had a good recovery without recurrent CAT or bleeding.
\end{abstract}

Keywords: Heparin-induced thrombocytopenia (HIT); cancer-associated venous thromboembolism (CAT); anticoagulant; rivaroxaban

Submitted Jun 28, 2019. Accepted for publication Sep 24, 2019.

doi: $10.21037 /$ tcr.2019.09.55

View this article at: http://dx.doi.org/10.21037/tcr.2019.09.55

\section{Introduction}

Venous thromboembolism (VTE) is a common and potentially life-threatening complication in cancer patients, with a greater risk than non-cancer patients (1). Furthermore, these specific patients may receive chemotherapy, glucocorticoid, or antiangiogenic agents during the course of their diseases, which consequently increase the risk of VTE. At present, anticoagulant therapy with low molecular weight heparin (LMWH) is suggested as the first-line treatment for cancer-associated venous thromboembolism (CAT) while rivaroxaban is considered as an alternative to patients without gastrointestinal cancer (2). Whereas, for certain special clinical scenarios, such as the patient suffered from heparin-induced thrombocytopenia (HIT), the optimal anticoagulant strategy poses a challenging task in these fragile patients. During the past decade, direct oral anticoagulants (DOACs) have been proven to be more effective or at least non-inferior to conventional anticoagulants [vitamin $\mathrm{K}$ antagonists (VKAs) and $\mathrm{LMWH}$ ] in terms of prophylaxis/treatment for VTE, with a lower risk for major bleeding (3). Here, we report a lung adenocarcinoma patient who suffered from a HIT. Finally, an individual DOACs, rivaroxaban, was chosen as an optimal anticoagulant in consideration of up-to-date evidence and patient's characteristics.

\section{Case presentation}

A 66-year-old male (weight: $52 \mathrm{~kg}$ ) was admitted with a diagnosis of lung adenocarcinoma (T4N0M1, stage IV), with the negative expression of EGFR and ALK gene. The patient was treated with first-line chemotherapy, including cisplatin $\left(75 \mathrm{mg} / \mathrm{m}^{2}\right)$, pemetrexed $\left(500 \mathrm{mg} / \mathrm{m}^{2}\right)$, and bevacizumab $(7.5 \mathrm{mg} / \mathrm{kg})$ for every 3 weeks. After the $3^{\text {rd }}$ course of chemotherapy, B-ultrasound revealed the presence of right internal jugular VTE (Figure 1). Laboratory tests showed the increase of serum $\mathrm{D}$-dimer value $(3.87$, reference range, $0-0.5 \mu \mathrm{g} / \mathrm{mL}$ ), meanwhile the 


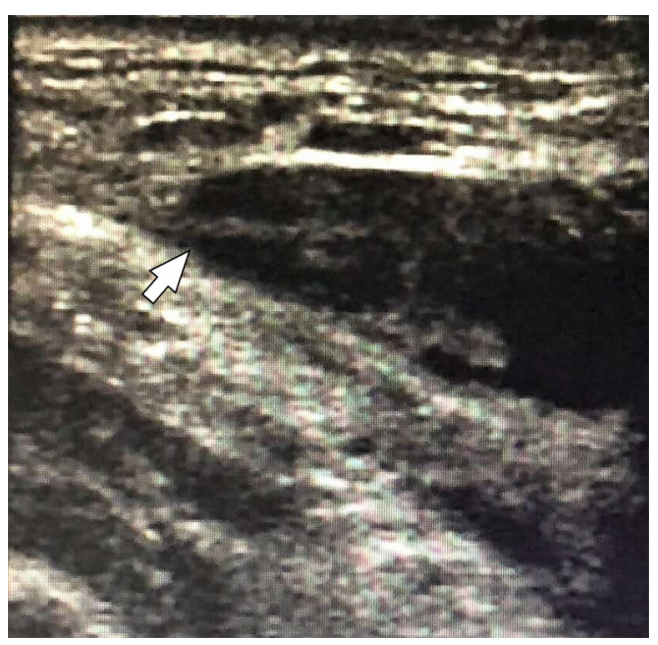

Figure 1 B-ultrasound revealed the right internal jugular VTE (arrow). VTE, venous thromboembolism.

Table 14 Ts score in this HIT patient

\begin{tabular}{ll}
\hline Items & Points \\
\hline Thrombocytopenia & $2(>50 \%$ fall and platelet nadir \\
& $\left.\geq 20 \times 10^{9} / \mathrm{L}\right)$ \\
Timing of platelet count fall or & 2 (clear onset between days 5 \\
other sequelae & and 10$)$ \\
Thrombosis or other sequelae & 0 (none) \\
Other cause for & 2 (no other cause for platelet \\
thrombocytopenia not evident & count fall is evident)
\end{tabular}

The pretest probability score: $6-8$, high probability. HIT, heparininduced thrombocytopenia.

platelet count was normal $\left(266 \times 10^{9} / \mathrm{L}\right)$ at this time. Therefore, nadroparin (4,100 IU twice daily, subcutaneous injection) was immediately administrated for the treatment of CAT. Five days later, the patient experienced a persisted epistaxis, and laboratory detection showed the serious thrombocytopenia $\left[(58-98) \times 10^{9} / \mathrm{L}\right]$. At this juncture, local compression is done for epistaxis. As a high 4Ts score (Table 1) (6 points, 2 points for platelet count fall $>50 \%$ and platelet nadir $\geq 20 \times 10^{9} / \mathrm{L}, 2$ points for timing of platelet count fall clear onset between day 5 and 10,2 points for no other cause for platelet count fall is evident) as well as the positive value of anti-platelet factor 4 (PF4)/ heparin antibodies ( 0.67 optical density, negative reference range $0-0.399)$, this patient was diagnosed with HIT and thereafter LMWH therapy was replaced with rivaroxaban $(15 \mathrm{mg}$ twice daily at beginning and $20 \mathrm{mg}$ once daily after 21 days) (4). On the $12^{\text {th }}$ day of rivaroxaban therapy, platelet count showed a good recovery $\left(229 \times 10^{9} / \mathrm{L}\right)$. During three months follow-up, the patient has been doing well without any evidence of recurrent CAT and bleeding, and the repeat platelet count was stable $\left[(190-266) \times 10^{9} / \mathrm{L}\right]$.

\section{Discussion}

Cancer patients, due to both disease and corresponding therapy, were considered to have a greater risk of VTE when compared to patients without cancer. For these fragile population, the Khorana risk score, which includes site of cancer, platelet count, total leukocytes, hemoglobin concentration and body mass index (BMI), can help us to identify the high thrombotic risk patients (5). In this case, the Khorana risk score was 1 for the site of cancer, which meant that the rate of CAT was $1.8-2.0 \%$ with intermediate-risk.

Bevacizumab, as a humanized monoclonal neutralizing antibody against vascular endothelial growth factor (VEGF), was considerably proved to provide a significant survival advantage in the treatment of non-small cell lung cancer (NSCLC) as an addition to platinum-based chemotherapy (6). Whereas, the mechanism of VEGF-antibody reminds us that the use of bevacizumab may cause serious thrombosis-associated clinical events, such as VTE and stroke. A meta-analysis including 13,185 patients showed an increased risk for venous thromboembolic events associated with bevacizumab use in cancer patients (7). In the present patient, bevacizumab usage also contributes to the development of CAT.

Current guidelines recommended the continued LMWH therapy (at least 6 months) for acute CAT (8). This drug strategy comes mainly from the CLOT study, which demonstrated a statistically significant reduction in recurrent VTE and improvement in survival with LMWH versus oral anticoagulation (OAC) in patients with CAT (9). In this case, patient received standard therapy by nadroparin (LMWH). Unfortunately, serious thrombocytopenia occurred and is considered as HIT according to 4Ts score criterion and $\mathrm{PF} 4$ value.

HIT, occurring in anticoagulation therapy with heparin or $\mathrm{LMWH}$, can be categorized as the nonimmunogenic form (type 1) with a benign course and immune-mediated form (type 2) which is a life-threatening adverse drug reaction (10). Clinically, the incidence of HIT type 2 relative to $\mathrm{LMWH}$ is rare $(0.1-0.2 \%$ in different studies) $(11,12)$. HIT is caused by the development of heparindependent $\operatorname{IgG}$ antibodies directed against a complex of PF4 by exposure to heparin $(13,14)$. Guidelines recommend that patients with HIT should cease heparin or LMWH 
therapy and prompt initiation of anticoagulation with argatroban, bivalirudin or fondaparinux (15). Nevertheless, these agents are burdensome because they require parenteral administration and frequent laboratory monitoring to adjust dosage, which may limit their long-term adoption. Given above limitations, oral anticoagulants (warfarin or DOACs) may represent an alternative choice for this patient. For cancer patients taking warfarin, time in therapeutic range (TTR) is hard to be well-controlled owing to frequent interactions with chemotherapeutic agents and immunosuppressive agents in anticancer therapy. In addition, warfarin may increase the risk of venous limb gangrene and skin necrosis in the HIT patients in its initiation (16).

Other oral anticoagulants should be considered in such patient. Of late years, DOACs, with a predictable dose response and no need for laboratory monitoring, have been considered non-inferior and probably safer than VKAs in patients with VTE. Whereas, evidence of DOACs on the treatment of CAT was limited, and guidelines' recommendations of DOACs treatment for CAT was only class IIa level C (2). Encouragingly, several clinical trials of DOACs that specially aimed at patients with cancer have been finished. Hokusai-Cancer study, which included 1050 patients with cancer and VTE, showed that the use of edoxaban (Xa factor inhibitor) for up to 12 months reduced the risk of recurrent VTE but increased risk of major bleeding when compared to dalteparin (17). SELECT-D trial also observed that rivaroxaban (Xa factor inhibitor) was associated with relatively low VTE recurrence but higher clinical relevant nonmajor bleeding (CRNMB) compared with dalteparin in cancer patients with VTE (18). The latest AVERT trial, which aimed at ambulatory patients with cancer who were at intermediate-to-high risk for VTE (Khorana score $\geq 2$ ) and were initiating chemotherapy, suggested a significantly lower risk of VTE and a higher risk of major bleeding for the use of apixaban when compared to the use of placebo (19). Accordingly, treatment with DOACs in cancer patients may reduce the risk of VTE at the expense of increased risk of bleeding. Thus, the net clinical benefit (NCB) is of concern, which has been growingly used to quantify both thromboembolism and hemorrhage in the field of anticoagulant treatment. Our prior NCB analysis supported that DOACs might represent a better NCB property compared to VKA and LWMHs in patients with cancer (20). On the basis of available evidence, DOACs are likely to be a reasonable alternative for CAT patients who are unable to use LWMHs. Regarding individual DOACs, only rivaroxaban, which was approved for treating VTE by China
Food and Drug Administration (CFDA), has been evaluated on the efficacy and safety in patients with HIT in prospective study. In this study, none of the treated patients experienced any major bleeding and platelet recovery was achieved in all patients who completed treatment, which showed rivaroxaban appear to be a treatment option in HIT patients (21). To summarize, rivaroxaban might be an effective and safe agent to treat with CAT patients who suffered from HIT. In our case, the patient was followed by rivaroxaban over 3 months, without recurrent VTE and no episodes of bleeding.

In conclusion, based on current evidence, we believe that rivaroxaban use has numerous advantages in this clinical scenario, including patient compliance, easy to access, and positive NCB property. However, further studies with large sample size on evaluation of DOACs in the treatment of these fragile population are necessary.

\section{Acknowledgments}

Funding: The present Case Report was supported by Research Funds of Shanghai Health and Family Planning commission (20184Y0022), Cultivation fund of clinical research of Renji hospital (PY2018-III-06), Clinical Pharmacy Innovation Research Institute of Shanghai Jiao Tong University School of Medicine (CXYJY2019ZD001, CXYJY2019QN004) and Program for Key but Weak Disciplines of Shanghai Municipal Commission of Health and Family Planning (2016ZB0304).

\section{Footnote}

Conflicts of Interest: The authors have completed the ICMJE uniform disclosure form (available at http://dx.doi. org/10.21037/tcr.2019.09.55). The authors have no conflicts of interest to declare.

Ethical Statement: The authors are accountable for all aspects of the work in ensuring that questions related to the accuracy or integrity of any part of the work are appropriately investigated and resolved. All procedures performed in studies involving human participants were in accordance with the ethical standards of the institutional and/or national research committee(s) and with the Helsinki Declaration (as revised in 2013). Written informed consent was obtained from the patient for publication of this manuscript and any accompanying images.

Open Access Statement: This is an Open Access article 
distributed in accordance with the Creative Commons Attribution-NonCommercial-NoDerivs 4.0 International License (CC BY-NC-ND 4.0), which permits the noncommercial replication and distribution of the article with the strict proviso that no changes or edits are made and the original work is properly cited (including links to both the formal publication through the relevant DOI and the license). See: https://creativecommons.org/licenses/by-nc-nd/4.0/.

\section{References}

1. Blom JW, Vanderschoot JP, Oostindiër MJ, et al. Incidence of venous thrombosis in a large cohort of 66,329 cancer patients: results of a record linkage study. J Thromb Haemost 2006;4:529-35.

2. Konstantinides SV, Meyer G, Becattini C, et al. 2019 ESC Guidelines for the diagnosis and management of acute pulmonary embolism developed in collaboration with the European Respiratory Society (ERS). Eur Heart J 2019. [Epub ahead of print].

3. Kohn CG, Lyman GH, Beyer-Westendorf J, et al. Effectiveness and safety of rivaroxaban in patients with cancer-associated venous thrombosis. J Natl Compr Canc Netw 2018;16:491-7.

4. Lo GK, Juhl D, Warkentin TE, et al. Evaluation of pretest clinical score (4 T's) for the diagnosis of heparin-induced thrombocytopenia in two clinical settings. J Thromb Haemost 2006;4:759-65.

5. Khorana AA, Kuderer NM, Culakova E, et al. Development and validation of a predictive model for chemotherapyassociated thrombosis. Blood 2008;111:4902-7.

6. Qu CY, Zheng Y, Zhou M, et al. Value of bevacizumab in treatment of colorectal cancer: a meta-analysis. World J Gastroenterol 2015;21:5072-80.

7. Alahmari AK, Almalki ZS, Alahmari AK, et al. Thromboembolic events associated with bevacizumab plus chemotherapy for patients with colorectal cancer: a metaanalysis of randomized controlled trials. Am Health Drug Benefits 2016;9:221-32.

8. Kearon C, Akl EA, Ornelas J, et al. Antithrombotic therapy for VTE disease: CHEST guideline and expert panel report. Chest 2016;149:315-52.

9. Lee AY, Rickles FR, Julian JA, et al. Randomized comparison of low molecular weight heparin and coumarin derivatives on the survival of patients with cancer and venous thromboembolism. J Clin Oncol 2005;23:2123-9.

10. Martel N, Lee J, Wells PS. Risk for heparin-induced thrombocytopenia with unfractionated and low-molecular- weight heparin thromboprophylaxis: a meta-analysis. Blood 2005;106:2710-5.

11. Smythe MA, Koerber JM, Mattson JC. The incidence of recognized heparin-induced thrombocytopenia in a large, tertiary care teaching hospital. Chest 2007;131:1644-9.

12. Warkentin TE, Sheppard JA, Horsewood P, et al. Impact of the patient population on the risk for heparin-induced thrombocytopenia. Blood 2000;96:1703-8.

13. Warkentin TE, Levine MN, Hirsh J, et al. Heparininduced thrombocytopenia in patients treated with lowmolecular-weight heparin or unfractionated heparin. $\mathrm{N}$ Engl J Med 1995;332:1330-5.

14. Watson H, Davidson S, Keeling D, et al. Guidelines on the diagnosis and management of heparin-induced thrombocytopenia: second edition. Br J Haematol 2012;159:528-40.

15. Cuker A, Arepally GM, Chong BH, et al. American Society of Hematology 2018 guidelines for management of venous thromboembolism: heparin-induced thrombocytopenia. Blood Adv 2018;2:3360-92.

16. Liebman HA. Heparin-induced thrombocytopenia: diagnosis and management. Vascular 2008;16 Suppl 1:S71-6.

17. Raskob GE, van Es N, Verhamme P, et al. Edoxaban for the treatment of cancer-associated venous thromboembolism. N Engl J Med 2018;378:615-24.

18. Young AM, Marshall A, Thirlwall J, et al. Comparison of an oral factor Xa inhibitor with low molecular weight heparin in patients with cancer with venous thromboembolism: results of a randomized trial (SELECT-D). J Clin Oncol 2018;36:2017-23.

19. Carrier M, Abou-Nassar K, Mallick R, et al. Apixaban to Prevent Venous Thromboembolism in Patients with Cancer. N Engl J Med 2019;380:711-9.

20. Yan YD, Zhang C, Shen L, et al. Net Clinical benefit of non-vitamin $\mathrm{K}$ antagonist oral anticoagulants for venous thromboembolism prophylaxis in patients with cancer: a systematic review and trade-off analysis from 9 randomized controlled trials. Front Pharmacol 2018;9:575.

21. Linkins LA, Warkentin TE, Pai M, et al. Rivaroxaban for treatment of suspected or confirmed heparin-induced thrombocytopenia study. J Thromb Haemost 2016;14:1206-10.

Cite this article as: Le KJ, Yan YD, Liu YX, Xu JB, Cui $M$, Gu ZC. Rivaroxaban treatment for cancer-associated venous thromboembolism in a patient with heparininduced thrombocytopenia: a case report. Transl Cancer Res 2019;8(6):2481-2484. doi: 10.21037/tcr.2019.09.55 